(Aus dem pharmakologischen Institut der Universität Tübingen.)

\title{
Beiträge zur Frage über die Wirkung der Xanthinderivate.
}

II. Mitteilung.

Über die Veränderung mechanischer Eigenschaften des ruhenden quergestreiften Muskels (Froschgastrocnemius) unter dem Einflusse der versehieden alkylierten Xanthine.

Von

Dr. med. J. W. Golowimski,

Assistent am physiologischen Institut der Universität Moskau.

Nachdem wir in der vorigen Mitteilung die elastischen Eigenschaften des ruhenden quergestreiften Muskels kennen gelernt haben, können wir uns nun der wichtigen Frage zuwenden, $o b$ und in welcher Weise pharmakologische Agentien imstande sind, diese elastischen Eigenschaften des ruhenden Muskels zu verändern.

Die in der Literatur über diese Frage bereits vorliegenden Untersuchungen [Rossbach und Anrep ${ }^{1}$ ), Kobert ${ }^{2}$ ), Dreser ${ }^{3}$ ), Goto $\left.{ }^{4}\right)$ u. a.] beweisen, dass die Muskelelastizität durch pharmakologische Agentien bedeutende Veränderungen erfahren kanı. Kobert hat durch seine Versuche festgestellt, dass bei grösseren Coffeindosen der quergestreifte Muskel seine Elastizität allmählich verliert. Von besonderem Werte sind die hierher gehörenden Untersuchungen Dreser's mit verschiedenen pharmakologischen Agentien (darunter auch dem Coffein), da hier eine Methode für die genaue mathematische Berechnung der quantitativen Abweichung von der Norm gegeben wird. Der Einfluss des Coffeins ist nach diesen

1) Pflüger's Arch. f. d. ges. Physiol. Bd. 21.

2) Arch. f. exper. Pathol. u. Pharmak. Bd. 15 S. 22-80. 1882.

3) Arch. f. exper. Pathol. u. Pharmak. Bd. 27 S. 50.

4) Zeitschr. f. Biol. Bd. 16 S. 38.1904. 
Versuchen sehr abhängig von der Dosis. Kleine Dosen (1-2 mg) erhöhen nach Dreser die Dehnbarkeit des ruhenden Muskels, grössere aber vermindern dieselbe, wobei die Dehnungskurve mehr geradlinig verläuft als beim normalen Muskel. Dreser stellte seine Versuche bei normaler Blutzirkulation in den Froschextremitäten an, führte die betreffenden Substanzen in den Bauchlymphsack ein und uahm die Beobachtungen 2-3 Stunden nach der Injektion auf. Ein so langes Abwarten kann für die Beurteilung der Resultate uicht gleichgültig sein. Einmal können bei solcher Art der Injektion die betreffenden Körper der Xanthingruppe mit allmählich zunebmender Ausbreitung lokale Wirkungen auch auf benachbarte Gewebe ausüben, und sodann muss wegen der ungleichmässigen Rescrption das quantitative Eintreten der Substanzen in das Blut grossen Schwankungen unterliegen.

Meine nachfolgenden Versuche über die Wirkung verschieden alkylierter Xanthine auf die elastischen Eigenschaften des ruhenden Muskels wurden mit dem bereits in der letzten Mitteilung erwäbnten Apparat von $\mathrm{C}$. Jacobj ${ }^{1}$ ) angestellt, und zwar bei völlig erhaltener Blutzirkulation. Um aber die oben erwähnten Fehlerquellen zu vermeiden, wurden die zu untersuchenden Substanzen nicht in den Bauchlymphsack, sondern direkt in die Bauchvene injiziert. Die Beobachtungen wurden erst 3 Minuten nach der Injektion aufgenommen. Es wurden stets Tiere einer Art (R. esculenta) und möglichst von gleichem Gewichte verwendet. Die Substanzen wurden proportional ihrem Gehalt an freier Base pro Gramm Körpergewicht des Tieres berechnet und injiziert. Um die nötige Bewegungslosigkeit der Frösche zu erzielen, wurden die motorischen Nerven der Extremitäten möglichst hoch durchschnitten.

Die untenstehenden Resultate geben die Berechnungen der Dehnungs- und Rückdehnungskurven des Froschgastrocnemius in der Norm und unter dem Einfluss der verschieden alkylierten Xanthine wieder. Diese Berechnungen erfolgten nach der von Dreser ${ }^{2}$ ) angegebenen Metbode; jedoch wurden die Arbeits- und Dreiecksflächen durch Wägen bestimmt, wobei die Fehlergrenzen zwischen $0,10-0,25 \%$ schwanken.

1) Deutsche med. Wochenschr. 1906 S. 1646 (Vortragsreferat.)

2) $1 . \mathrm{c}$. 


\section{Dehnungsversuche des ruhenden Froschgastrocnemius.}

Trimethylxanthinum.

Rana esculenta, t. $40,0 \mathrm{~g}$.

Norm . . . . . . . $\left\{\begin{array}{l}\text { Arbeitsfläche } \\ \text { Dreiecksfläche }\end{array}=\frac{0,1240}{0,1780}=69,6 \%\right.$.

$10^{-4}$ pro 1,0 des Körpergewichts $\left\{\frac{\text { Arbeitsfläche }}{\text { Dreiecksfläche }}=\frac{0,0971}{0,1310}=74,1 \%\right.$.

Äthyltheophyllinum.

Rana esculenta, o. $40,0 \mathrm{~g}$.

Norm . . . . . . $\left\{\frac{\text { Arbeitsfläche }}{\text { Dreiecksfäche }}=\frac{0,1263}{0,1782}=70,8 \%\right.$.

$10^{-4}$ pro 1,0 des Körpergewichts $\left\{\frac{\text { Arbeitsfläche }}{\text { Dreiecksfläche }}=\frac{0,1761}{0,2351}=74,9 \%\right.$.

Äthyltheobrominum.

Rana esculenta, t. $35,0 \mathrm{~g}$.

Norm . . . . . . . $\left\{\frac{\text { Arbeitsfläche }}{\text { Dreiecksfläche }}=\frac{0,1110}{0,1690}=65,6 \%\right.$.

$10^{-4}$ pro 1,0 des Körpergewichts $\left\{\frac{\text { Arbeitsfläche }}{\text { Dreiecksfläche }}=\frac{0,1450}{0,2080}=69,7 \%\right.$

Äthylparaxanthinum.

Rana esculenta, む. $40,0 \mathrm{~g}$.

Norm . . . . . $\left\{\frac{\text { Arbeitsfläche }}{\text { Dreiecksfläche }}=\frac{0,0986}{0,1615}=61,0{ }^{\circ} \%\right.$

$10^{-4}$ pro 1,0 des Körpergewichts $\left\{\frac{\text { Arbeitsfläche }}{\text { Dreiecksfläche }}=\frac{0,1360}{0,2067}=65,7 \%\right.$.

Theobrominum.

Rana esculenta, t. $40,0 \mathrm{~g}$.

Norm . . . . . . $\left\{\begin{array}{l}\text { Arbeitsfläche } \\ \text { Dreiecksfläche }\end{array}=\frac{0,1005}{0,1755}=57,1 \%\right.$.

$10^{-4}$ pro 1,0 des Körpergewichts $\left\{\frac{\text { Arbeitsfläche }}{\text { Dreiecksfläche }}=\frac{0,1240}{0,1940}=63,9 \%\right.$.

Theophyllinum.

Rana esculenta, o. 40,0 g.

Norm . . . . . $\left\{\frac{\text { Arbeitsfläche }}{\text { Dreiecksfläche }}=\frac{0,1350}{0,2002}=67,4 \%\right.$.

$10^{-4}$ pro 1,0 des Körpergewichts $\left\{\frac{\text { Arbeitsfläche }}{\text { Dreiecksfläche }}=\frac{0,1971}{0,2633}=74,8 \%\right.$. 


\section{Paraxanthinum.}

Rana esculenta, ঠ. $35,0 \mathrm{~g}$.

Norm . . . . . . $\left\{\frac{\text { Arbeitsfläche }}{\text { Dreiecksfläche }}=\frac{0,0991}{0,1651}=60,0 \%\right.$. $10^{-4}$ pro 1,0 des Körpergewichts $\left\{\frac{\text { Arbeitsfläche }}{\text { Dreiecksfläche }}=\frac{0,1480}{0,2200}=67,2 \%\right.$.

Heteroxanthinum.

Rana esculenta, む. $35,0 \mathrm{~g}$.

Norm . . . . . $\left\{\frac{\text { Arbeitsfläche }}{\text { Dreiecksfläche }}=\frac{0,0982}{0,1625}=60,4 \%\right.$. $10^{-4}$ pro 1,0 des Körpergewichts $\left\{\frac{\text { Arbeitsfläche }}{\text { Dreiecksfläche }}=\frac{0,1185}{0,1710}=69,2 \%\right.$.

Methoxycoffeinum.

Rana esculenta, $\delta . ~ 35,0 \mathrm{~g}$.

Norm . . . . . $\left\{\begin{array}{l}\text { Arbeitsfläche } \\ \text { Dreiecksfläche }\end{array}=\frac{0,1200}{0,1940}=62,8 \%\right.$. $10^{-4}$ pro 1,0 des Körpergewichts $\left\{\frac{\text { Arbeitsfläche }}{\text { Dreiecksfläche }}=\frac{0,1160}{0,1670}=66,4 \%\right.$.

Äthoxycoffeinum.

Rana esculenta, ঠ. 36,0 g.

Norm . . . . . . $\left\{\frac{\text { Arbeitsfläche }}{\text { Dreiecksfläche }}=\frac{0,1251}{0,1911}=65,9 \%\right.$. $10^{-4}$ pro 1,0 des Körpergewichts $\left\{\frac{\text { Arbeitsfläche }}{\text { Dreiecksfl̈̈che }}=\frac{0,1300}{0,1890}=68,7 \%\right.$.

\section{Riickdehnungsversuche des ruhenden Froschgastrocnemius.}

Paraxanthinum.

Rana esculenta, t. 40,0 g.

Norm . . . . . . $\left\{\frac{\text { Arbeitsfläche }}{\text { Dreiecksfläche }}=\frac{0,1021}{0,1781}=57,3 \%\right.$. $10^{-4}$ pro 1,0 des Körpergewichts $\left\{\frac{\text { Arbeitsfläche }}{\text { Dreiecksfläche }}=\frac{0,0870}{0,1740}=50,0 \%\right.$.

Theobrominum.

Rana esculenta, ঠ. $40,0 \mathrm{~g}$. Norm . . . . . . $\left\{\frac{\text { Arbeitsfläche }}{\text { Dreiecksfläche }}=\frac{0,0870}{0,1691}=51,4 \%\right.$. $10^{-4}$ pro 1,0 des Körpergewichts $\left\{\frac{\text { Arbeitsfläche }}{\text { Dreiecksfläche }}=\frac{0,0871}{0,1981}=43,9 \%\right.$. 
Theophyllinum.

Rana esculenta, t. 38,0 g.

Norm . . . . . . $\left\{\frac{\text { Arbeitsfläche }}{\text { Dreiecksfläche }}=\frac{0,0970}{0,1900}=51,0 \%\right.$. $10^{-4}$ pro 1,0 des Körpergewichts $\left\{\begin{array}{l}\text { Arbeitsfläche } \\ \text { Dreiecksfläche }\end{array}=\frac{0,0880}{0,1950}=45,1 \%\right.$.

\section{Coffeinum.}

Rana esculenta, む. 40,0 g. Norm . . . . . . $\left\{\begin{array}{l}\text { Arbeitsfläche } \\ \text { Dreiecksfläche }\end{array}=\frac{0,0930}{0,1870}=49,7 \%\right.$. $10^{-4}$ pro 1,0 des Körpergewichts $\left\{\begin{array}{l}\text { Arbeitsfläche } \\ \text { Dreiecksfläche }\end{array}=\frac{0,0990}{0,2131}=46,4 \%\right.$.

Äthyltheophyllinum.

Rana esculenta, t. 36,0 g.

Norm . . . . . . . $\left\{\frac{\text { Arbeitsfläche }}{\text { Dreiecksfläche }}=\frac{0,1030}{0,1980}=52,0 \%\right.$. $10^{-4}$ pro 1,0 des Körpergewichts $\left\{\frac{\text { Arbeitsfläche }}{\text { Dreiecksfläche }}=\frac{0,0880}{0,1820}=48,3 \%\right.$.

Äthyltheobrominum.

Rana esculenta, ठ. $40,0 \mathrm{~g}$. Norm . . . . . . . $\left\{\begin{array}{l}\text { Arbeitsfläche } \\ \text { Dreiecksfläche }\end{array}=\frac{0,1240}{0,2150}=57,6 \%\right.$. $10^{-4}$ pro 1,0 des Körpergewichts $\left\{\frac{\text { Arbeitsfläche }}{\text { Dreiecksfläche }}=\frac{0,1460}{0,2720}=53,6 \%\right.$. Äthylparaxanthinum.

Rana esculenta, ঠ. 39,0 g. Norm . . . . . . $\left\{\frac{\text { Arbeitsfläche }}{\text { Dreiecksfläche }}=\frac{0,0986}{0,1971}=50,0 \%\right.$. $10^{-4}$ pro 1,0 des Körpergewichts $\left\{\begin{array}{l}\text { Arbeitsfläche } \\ \text { Dreiecksfläche }\end{array}=\frac{0,0800}{0,1731}=46,2 \%\right.$.

Heteroxanthinum.

Rana esculenta, ‡. 35,0 g. Norm . . . . . . $\left\{\frac{\text { Arbeitsfläche }}{\text { Dreiecksfläche }}=\frac{0,0884}{0,1631}=54,1 \%\right.$. $10^{-4}$ pro 1,0 des Körpergewichts $\left\{\frac{\text { Arbeitsfläche }}{\text { Dreiecksfläche }}=\frac{0,0891}{0,1891}=47,1 \%\right.$. 


\section{Methoxycoffeinum.}

Rana esculenta, む. $35,0 \mathrm{~g}$.

Norm . . . . . . $\left\{\frac{\text { Arbeitsfläche }}{\text { Dreiecksfläche }}=\frac{0,1160}{0,2390}=48,5 \%\right.$. $10^{-4}$ pro 1,0 des Körpergewichts $\left\{\frac{\text { Arbeitsfläche }}{\text { Dreiecksfläche }}=\frac{0,0830}{0,1830}=45,3 \%\right.$.

Äthoxycoffeinum.

Rana esculenta, む. $40,0 \mathrm{~g}$.

Norm . . . . . . $\left\{\frac{\text { Arbeitsfläche }}{\text { Dreiecksfläche }}=\frac{0,0940}{0,1800}=52,2 \%\right.$.

$10^{-4}$ pro 1,0 des Körpergewichts $\left\{\frac{\text { Arbeitsfläche }}{\text { Dreiecksfläche }}=\frac{0,1161}{0,2360}=49,1 \%\right.$.

Tabelle.

\begin{tabular}{|c|c|c|c|}
\hline Substanzen & Dosis & $\begin{array}{l}\text { Verminderung der } \\
\text { elastischen Dehnbar- } \\
\text { keit des Frosch- } \\
\text { gastrocnemius } \\
\text { in Prozenten }\end{array}$ & $\begin{array}{l}\text { Unvollkommenheit d. } \\
\text { Energierestituierung } \\
\text { bei Rückdehnung d. } \\
\text { Froschgastrocnemius } \\
\text { in Prozenten }\end{array}$ \\
\hline 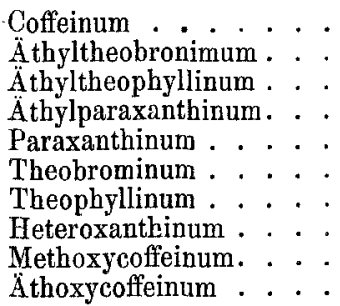 & 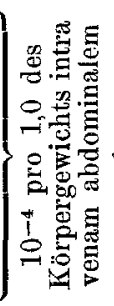 & $\begin{array}{r}6,4 \\
6,2 \\
5,7 \\
7,7 \\
12,0 \\
11,9 \\
10,9 \\
12,8 \\
5,7 \\
5,0\end{array}$ & $\begin{array}{r}6,6 \\
7,2 \\
7,1 \\
7,6 \\
12,7 \\
12,6 \\
11,5 \\
12,9 \\
6,5 \\
5,9\end{array}$ \\
\hline
\end{tabular}

Aus diesen Versuchsergebnissen folgt, dass die Xanthine nicht ohne Einfluss auf die elastischen Eigenschaften des ruhenden Muskels bleiben. Der Charakter der Dehnungskurve bleibt überall gleich, aber die Dehnbarkeit vermindert sich, und die Dehnungskurve nähert sich der geraden Linie. Diese Beobachtungen stimmen vollkommen überein mit den Angaben D reser's ${ }^{1}$ ) bei mittleren Coffeindosen.

Der Unterschied in der Wirkung der einzelnen von mir geprüften Xanthinkörper ist lediglich ein quantitativer.

Ausser der Dehnungskurve verändert sich, wie aus den vorstehenden Berechnungen hervorgeht, auch die der Rückdehnung, die unter der Einwirkung der Xanthine noch unvollkommener wird.

1) 1. c. 
Alle diese Erscheinungen sind im hohen Grade abhängig von dem Einfluss der Substanzen dieser Gruppe auf die kontraktile Muskelsubstanz, die sich in folgenden Veränderungen ausdrückt: Das allererste Stadium der Wirkung äussert sich in einer Kondensierung der Eiweissmoleküle; erst nach dieser wird eine feinere Granulierung in der Struktur bemerkbar, die sich durch Trübung charakterisiert und eine Folge beginnender Gerinnung des Myosins darstellt; endlich tritt dann eine vollständige Vernichtung und Zerstörung des Muskelbaues ein. Für kleine und mittlere Dosen kommen nur die ersten beiden Stadien der Wirkung in Betracht, die als reversible Prozesse aufzufassen sind, d. h. nach dem Aufhören der Wirkung kehrt die Struktur zur Norm zurück.

Die bei der Wirkung der Xanthinkörper beobachtete Verminderung der Dehnbarkeit des Muskels ist danach ganz verständlich; ihre Entstehungsweise zeigt eine weitgehende Analogie zu der Wirkung, wie man sie bei der Tetanisierung des Muskels beobachtet. In beiden Fällen handelt es sich in der Tat um eine Verkürzung einzelner Muskelfasern, und infolgedessen beteiligt sich der Muskel einheitlicher mit seinen Bestandelementen bei der Dehnung.

Ein Unterschied besteht lediglich in der Wirkung auf die Rückdehnungskurve.

Unter der Einwirkung der Xanthinderivate beobachtet man, wie aus den Kurvenberechnungen klar hervorgeht, ein stärkeres Hervortreten der sogenannten elastischen Nachwirkung. Bekanntlich verwandelt sich nach den Untersuchungen von Fick ${ }^{1}$ ) bei der Dehnung des Muskels ein Teil der Arbeit in Wärme, und diese Umsetzung in Wärme kann unter dem spezifischen Einfluss der Xanthinkörper auf die kontraktile Muskelsubstanz in erhöhtem Maasse vor sich gehen, ähnlich wie dies z. B. bei dem unter Veratrin-Einwirkung arbeitenden Muskel mit Hilfe thermoelektrischer Messungen von Fick und B oehm festgestellt ist. Tritt aber ein derart starker Energieverbrauch bei der Deformation des Muskels durch die Wirkung der Xanthinkörper ein, so kann er natürlich zu einer gegen die Norm unvollkommeneren Rückdehnungskurve führen; denn die in Wärme umgesetzte Energie

1) Untersuchungen aus dem physiol. Laboratorium der Züricher Hochschule Nr. 1. Wien 1869. - Mechanische Arbeit und Wärmeentwicklung bei der Muskeltätigkeit. Leipzig 1882. 
muss nach dem Gesetz der Erhaltung der Energie von der Muskelarbeit entnommen werden.

Bezüglich des Wirkungsgrades der Xanthinkörper auf die elastischen Eigenschaften des ruhenden Muskels ist zu bemerken: Am schwächsten wirken die trialkylierten Xanthine, etwas stärker ist die Wirkung bei den Dimethylxanthinen, und am stärksten tritt sie hervor beim Monomethylxanthin-Heteroxanthin; $d$. h. je weniger der Xanthinkern der Alkylierung unterworfen wird, um so schärfer tritt der Einfluss auf die Elastizität des Muskels hervor.

Zieht man nun den chemischen Aufbau 'der Purinkörper in Betracht, um die Bedeutung der Methyl- (resp. Methoxy-) oder Äthyl(resp. Äthoxy-) Gruppen in dieser Hinsicht zu erklären, so erweist sich, dass ein Ersatz des Methyls beim N durch Äthyl die Wirksamkeit des Xanthinkernes gar nicht oder doch höchstens in ganz geringem Grade verändert. Vergleicht man z. B. das Methyltheobromin mit dem Äthyltheobromin, so erhält man einen Unterschied von $0,2-0,6 \%$, der ohne Zweifel in den Grenzen des Beobachtungsfehlers liegen kann. Ähnliche Resultate gibt auch ein Vergleich der Wirksamkeit des methoxylierten und äthoxylierten Coffeins.

Eine gewisse Abstufung des Wirkungsgrades ist zu bemerken bei Lageveränderung der Methyl- und Äthylgruppen in den isomeren Verbindungen: Äthyltheophyllin, Äthyltheobromin, Äthylparaxanthin, Theophyllin, Theobromin, Paraxanthin, indem die Wirkung von Theophyllin zum Paraxanthin zunimmt, wogegen die Wirkung des Coffeins infolge Anlagerung der Methoxy- und Äthoxy-Gruppe an den C abnimmt. Immerhin handelt es sich auch hier nicht um grosse Unterschiede.

Es ist mir eine angenehme Pflicht, zum Schlusse Herrn Prof. C. Jacobj für die Anregung zu dieser Arbeit und seine vielseitige Unterstützung bei ihrer Anfertigung meinen herzlichsten Dank zu sagen. 